\title{
Radiological Imaging of the Small Bowel
}

\author{
A.G. Schreyer C. Stroszczynski \\ Department of Radiology, University Medical Center Regensburg, Regensburg, Germany
}

\author{
Key Words \\ Small bowel $\cdot$ Magnetic resonance imaging . \\ Computed tomography $\cdot$ Small bowel diagnosis
}

\begin{abstract}
Recently introduced endoscopy-based imaging methods such as double-balloon endoscopy or wireless capsule endoscopy can visualize the complete small bowel. These approaches are quite invasive diagnostic methods. Therefore, radiological small bowel imaging is also still considered as the primary imaging approach to diagnose pathological changes of the small bowel. In this review article the most important small bowel imaging modalities such as conventional fluoroscopy, computed tomography, magnetic resonance imaging and ultrasound are discussed. Additionally the most important diseases, which can affect the small bowel, are evaluated and the optimal imaging modalities are pointed out, respectively.

Copyright $\odot 2011$ S. Karger AG, Basel
\end{abstract}

\section{Introduction}

Before the introduction of new endoscopy-based methods such as wireless capsule endoscopy, push enteroscopy and double-balloon endoscopy, the radiological approach was the only imaging method for the small bowel [1-3]. For small bowel diagnosis there are currently several radiological, endoscopic and scintigraphic ap- proaches. In this article we will focus on the radiological imaging methods providing a systematic review of the different modalities followed by the most important clinical indications for small bowel imaging including the radiological imaging recommendations.

\section{Conventional X-Ray and Fluoroscopy}

The most basic test for abdominal and small bowel imaging represents the conventional plain abdominal x-ray examination, which just allows the diagnosis of an ileus or the detection of free abdominal air. There is no further use for dedicated small bowel imaging or more subtle differential diagnosis [4].

The small bowel follow-through examination represents basically a fluoroscopically performed examination with the oral application of a positive intraluminal contrast medium such as barium or iodine-based contrast medium. The patient is asked to drink the contrast medium while a series of plain abdominal films are obtained. The technique is more suitable to detect a functional stenosis or to evaluate the passage time through the small bowel. This technique was mostly used in the USA and had quite similar results for detecting pathological changes of the small bowel, when performed by an experienced radiologist, compared to the enteroclysis of the small bowel using a double contrast, which represents the European approach [5-7]. For conventional small bowel enteroclysis, a nasojejunal probe has to be positioned

\section{KARGER \\ Fax +4161306 1234 \\ E-Mail karger@karger.ch}

www.karger.com
(C) 2011 S. Karger AG, Basel

$0257-2753 / 11 / 0297-0022 \$ 38.00 / 0$

Accessible online at:

www.karger.com/ddi
Andreas G. Schreyer, MD, MBA

Department of Radiology, University Medical Center Regensburg

DE-93042 Regensburg (Germany)

Tel. +499419447418

E-Mail andreas.schreyer@klinik.uni-regensburg.de 
fluoroscopically at the area at the ligament of Treitz to avoid contrast reflux [8]. After this procedure, barium, followed by methylcellulosis as a negative contrast medium, has to be applied continuously. The small bowel follow-through as well as the enteroclysis only depict intraluminal pathology and can cause a radiation dose between 2 and $4 \mathrm{mSv}$ depending on the experience of the examiner [9].

\section{Computed Tomography}

Sectional imaging such as computed tomography (CT) allows a comprehensive depiction of pathological changes not restricted to intraluminal findings detected by conventional x-ray techniques. Therefore, CT imaging is considered the primary emergency imaging modality after an ultrasound examination to assess an acute abdominal situation. With the introduction of fast multidetector CT (MDCT) scanners the whole abdomen can be acquired within a breath-hold. Additionally, based on the high-resolution acquisition, arbitrary image planes can be calculated. To be able to assess the small bowel wall, the bowel lumen has to be distended adequately. The bowel wall can be evaluated after the intravenous administration of iodized contrast medium. To allow a better discrimination of the bowel lumen and the bowel wall, a neutral intraluminal contrast is preferable. Therefore, water in combination with a distending substance such as methylcellulosis or mannitol is the method of choice. The intraluminal contrast medium can be applied just orally, which results in a so-called CT enterography. Alternatively a duodenal probe can be placed after the pylorus to allow continuous small bowel distension. This method, which is called CT enteroclysis, allows a better and more subtle visualization of the bowel loops because of their better distension. On the other hand, the current literature does not really give persuading evidence for any clinical benefit of the CT enteroclysis compared with the enterography, because relevant wall thickening and contrast uptake as a sign for bowel wall inflammation can even be assessed on not optimally distended bowel loops $[10,11]$. In the USA the CT-based small bowel imaging is considered as the method of choice. Radiation between 10 and $16.1 \mathrm{mSv}$ caused by MDCT for abdominal examination should raise some concern as a primary imaging and follow-up modality, especially for the mostly young patients suffering from inflammatory bowel disease [3].

Radiological Imaging of the Small Bowel



Fig. 1. Patient with penetrating CD: axial contrast-enhanced fatsaturated $\mathrm{T}_{1}$-weighted image showing affected small bowel with mesenteric enhancement (arrows) as a sign for penetrating CD.

\section{Magnetic Resonance Imaging}

Because of the continuous evolution and technical developments in magnetic resonance imaging (MRI), abdominal MRI has become a more widely available and accepted technique in the last 10 years. Using fast gradients and even high-field equipment such as 3-Tesla scanners [12], small bowel imaging based on MRI data is currently even the accepted standard of reference as a base examination of the small bowel in patients with Crohn's disease (CD) that is supported by international and national guidelines [13]. For the intraluminal contrast the so-called 'dark lumen technique' is the accepted standard $[14,15]$. For the dark lumen MRI technique the bowel lumen is filled and distended by water mixed with methylcellulose or mannitol identical to CT imaging. Having an intravenously applied gadolinium-based contrast medium the bowel wall enhances approximately $60 \mathrm{~s}$ after contrast application. Following a combination of the dark signal of water in $\mathrm{T}_{1}$-weighted sequences with an enhanced bright bowel wall after contrast application, the term 'dark lumen' was created (fig. 1). As described for CT imaging, the intraluminal contrast can be applied orally resulting in MR enterography. Analogous to CT imaging, after a contrast application using a duodenal probe, which has to be placed fluoroscopically, the examination is called MR enteroclysis. Analogous to CT imaging, there are only a few studies that compare MR enterography and MR enteroclysis. In 2004, our group published a paper comparing both techniques with an

Dig Dis 2011;29(suppl 1):22-26 
additional conventional enteroclysis in 21 patients with known CD [10]. The MR enterography revealed in 6 of 21 patients more relevant pathological findings, while there was no difference between MR enterography and MR enteroclysis. Currently there is still no evidence-based recommendation for just oral or duodenal contrast application. We consider the strictly radiation-avoiding approach of MR enterography as the better examination with regard to the young age of most of the patients. Even if the images of MR enteroclysis 'look better' because of their better bowel distension, in most of the cases there will be no clinical benefit because relevant bowel wall thickening is visualized even in not perfectly distended bowel loops. Comparing MRI-based small bowel imaging with CT, the sensitivity for assessing bowel wall affection or abscesses in CD are similar [16].

The typical MR protocol consists of $\mathrm{T}_{1^{-}}$and $\mathrm{T}_{2}$-weighted sequences with intravenous contrast application. A study from 2002 found a sensitivity of just $43 \%$ based on single-shot sequences without contrast compared with a sensitivity of $93 \%$ with contrast-enhanced MRI [17]. The examination should be started with a fast and stable single-shot or single-slice sequence. $\mathrm{T}_{2}$-weighted single-shot spin-echo sequences such as HASTE (half Fourier acquired single-shot turbo spin echo) should be acquired to assess ascites as well as edema of the bowel wall. Additionally, gradient-echo sequences with a complete gradient inversion, which basically have a mixed signal with $\mathrm{T}_{2}$ predominance such as True-FISP (true fast imaging with steady precession), are helpful. For dark lumen imaging, $3 \mathrm{D}$ gradient-echo sequences such as VIBE (volume interpolated breath-hold examination) allow an excellent signal within a 3D dataset to assess the bowel wall. Adding $\mathrm{T}_{2}$-weighted high-resolution sequences in the pelvic fistulas can be depicted with an extremely good anatomical resolution.

\section{Ultrasound}

High-resolution ultrasound of the small bowel is considered as an emerging and accepted imaging method. The duodenum as well as jejunum and ileum can be depicted by ultrasound, although the complete small bowel cannot be depicted in most patients mostly because of an air artifact or superimposition of the small bowel loops. The terminal ileum as well as the appendix can be visualized in most cases. Using high-resolution probes between 4 and $10 \mathrm{MHz}$, even the small bowel stratification can be depicted. Increased vascularization as a consequence of inflammation can be seen using the power Doppler technique. Conventional B-mode ultrasound can exactly depict localization and length of affected segments and can simultaneously show complications such as fistulae, abscess and stenosis.

Contrast-enhanced ultrasound (CEUS) allows realtime visualization of gas-filled microbubbles with a diameter between 2 and $6 \mu \mathrm{m}$, which are surrounded by a shell composed of varying lipids and polymers. The advantage of CEUS over power Doppler ultrasound is the semiquantitative measurement of microperfusion [18]. A recently introduced imaging feature of CEUS is the depiction of a destroyed mucosal barrier which could be a diagnostic feature in graft-versus-host disease imaging [19]. A general problem of ultrasound is the dependency of the examiner's experience as well as the inability to screen the entire small bowel because of difficult-to-access anatomical areas or air superimposition [20].

\section{Clinical Indications for Radiological Small Bowel Imaging}

\section{Inflammatory Bowel Disease}

In $\mathrm{CD}$ patients the small bowel is affected in $70 \%$ while it represents the only area of affection in just $30 \%$. Generally, ultrasound represents a good primary examination method which can even depict further details applying advanced imaging techniques such as high-resolution ultrasound as well as CEUS. For the assessment of patients with $\mathrm{CD}$ or inflammatory bowel diseases it is the first imaging modality. Some guidelines still recommend a complete small bowel examination such as MRI- or CTbased imaging for patients having their primary diagnosis of CD $[13,21]$.

Because of the lack of ionizing radiation and the ability to depict the whole abdomen, small bowel imaging based on MRI such as MR enterography or MR enteroclysis are considered the modality. The CT-based method shows a similar sensitivity and specificity but can cause a major radiation dose in the mostly young patients. A recently published meta-analysis shows a sensitivity of between 82 and $100 \%$ with a specificity between 71 and $100 \%$ for MR enterography. In the same meta-analysis the sensitivity for high-resolution ultrasound was between 78 and $96 \%$ with a specificity between 67 and $100 \%$. Because of the potentially high radiation doses based on frequently performed CT examinations, MRI and ultrasound methods should be employed in patients with inflammatory bowel disease [22] (table 1). 


\section{Small Bowel Bleeding}

Less than $10 \%$ of intestinal bleedings originate from the small bowel [23]. Endoscopy (especially double-balloon enteroscopy as well as capsule endoscopy) is still the primary method of choice to diagnose small bowel bleedings. Continuous gastrointestinal bleeding without endoscopic diagnosis or therapy has to be evaluated using advanced imaging methods such as sectional imaging, scintigraphy or angiography. Conventional digital subtraction angiography can be relevant for bleedings of $>0.5 \mathrm{ml}$ blood per minute. On the other hand, certain bleedings can be embolized by angiography, even when there is a major risk of small bowel necrosis. ${ }^{99 \mathrm{~m}} \mathrm{Tc}$-marked erythrocytes can be helpful to reveal small bowel bleeding even at rates between 0.1 and $0.4 \mathrm{ml} / \mathrm{min}$. Because of the coarse resolution of scintigraphy, the exact localization of bleedings is sometimes difficult. Additionally there are false positive as well as false negative findings between 10 and $20 \%$.

MDCT allows scanning the whole abdomen employing different contrast phases (arterial, late phase) within several seconds resulting in a sensitivity of $90 \%$ and a specificity of $30 \%$. Because of the suboptimal patient monitoring and long examination protocols, MRI is less suited to assess bleedings in the mostly critically ill patients.

\section{Tumors}

Small bowel tumors are an extremely rare entity representing just $5 \%$ of all intestinal tumors. Next to small bowel metastasis, neuroendocrine cell tumors, adenocarcinomas, gastrointestinal stroma cell tumors and lymphomas are the most common tumors. Small bowel lymphomas represent another important entity of small bowel neoplasias. Basically, small bowel MRI represents the best radiological methods for tumor diagnosis, because it includes extraluminal structures and has the best soft tissue contrast.

\section{Sprue}

An advanced stage of sprue (gluten-sensitive enteropathy) can be detected using conventional fluoroscopic barium examinations [24] as well as sectional imaging [25]. The definite diagnosis is still based on endoscopy with consecutive biopsy and histology. MRI examinations play a major role in excluding or assessing the extent of T-cellassociated enteropathy as a differential diagnosis [26].

\section{Diverticula}

Diverticula of the small bowel can be found in $1-5 \%$ of all patients showing relevant complications in up to $10 \%$
Table 1. Imaging modalities for the most important pathological changes of the small bowel

\begin{tabular}{lllll}
\hline Small bowel pathology & $\begin{array}{l}\text { Fluo- } \\
\text { roscopy }\end{array}$ & CT & MRI & $\begin{array}{l}\text { Ultra- } \\
\text { sound }\end{array}$ \\
\hline Inflammatory bowel disease & + & + & ++ & ++ \\
Tumor & + & + & ++ & + \\
Sprue & + & 0 & + & + \\
Diverticula & + & + & ++ & + \\
Small bowel bleeding & 0 & ++ & + & 0
\end{tabular}

$0=$ No recommendation; $+=$ can be helpful; $++=$ recom mended radiological imaging.

of the cases. Their size can be between several millimeters up to several centimeters. They can be the cause for obstruction, bleedings and can be the focus for an abdominal abscess. In the duodenum, juxtapapillary diverticula can be the reason for gallbladder concrements. Small diverticula with diameters of just several millimeters can be difficult to detect by radiological methods. Duodenal diverticula in the peripancreatic region can be assessed by MRI and magnetic resonance cholangiopancreatography [27].

Meckel's diverticulum is a potential source of recurrent bleedings in the mostly young patients. Scintigraphic methods ( ${ }^{99 \mathrm{~m}} \mathrm{Tc}$-pertechnetate) are helpful in the presence of heterotopic gastric mucosa. Radiological methods consist basically of MRI or CT of the small bowel, but do generally have a low sensitivity in their detection rates.

\section{Conclusion}

Even the small bowel can be visualized increasingly by advanced and sometimes invasive endoscopic methods such as double-balloon endoscopy and push enteroscopy, and radiological techniques are still the methods of choice in most small bowel diseases. Next to high-resolution ultrasound, which represents an excellent primary examination for abdominal and small bowel pathologies, currently small bowel MRI should be the method of choice for further diagnostic work-up.

\section{Disclosure Statement}

The authors declare that no financial or other conflict of interest exists in relation to the content of the article. 


\section{References}

1 Schreyer AG, Debl K, Herfarth H: Indications for magnetic resonance imaging in internal medicine. When do we really need this technology? Internist (Berl) 2010;51:451462.

$>2$ Schreyer AG, Gölder S, Seitz J, Herfarth H: New diagnostic avenues in inflammatory bowel diseases. Capsule endoscopy, magnetic resonance imaging and virtual enteroscopy. Dig Dis 2003;21:129-137.

$>3$ Schreyer AG, Seitz J, Feuerbach S, Rogler G, Herfarth H: Modern imaging using computer tomography and magnetic resonance imaging for inflammatory bowel disease. Inflamm Bowel Dis 2004;10:45-54.

4 Maglinte DD, Kelvin FM, O’Connor K, Lappas JC, Chernish SM: Current status of small bowel radiography. Abdom Imaging 1996; 21:247-257.

$\checkmark 5$ Ott DJ, Chen YM, Gelfand DW, Van Swearingen F, Munitz HA: Detailed per-oral small bowel examination vs. enteroclysis. Part II Radiographic accuracy. Radiology 1985;155: 31-34.

6 Toms AP, Barltrop A, Freeman AH: A prospective randomised study comparing enteroclysis with small bowel follow-through examinations in 244 patients. Eur Radiol 2001;11:1155-1160.

$>7$ Kelvin FM, Maglinte DD: Enteroclysis or small bowel follow-through in Crohn's diseases? Gastroenterology 1998; 114:13491351.

$>8$ Sellink JL: Radiologic examination of the small intestine by duodenal intubation. Acta Radiol 1974; 15:318-332.

$>9$ Jaffe TA, Gaca AM, Delaney S, Yoshizumi TT, Toncheva G, Nguyen G, et al: Radiation doses from small-bowel follow-through and abdominopelvic MDCT in Crohn's disease. AJR Am J Roentgenol 2007;189:1015-1022.

10 Schreyer AG, Geissler A, Albrich H, Scholmerich J, Feuerbach S, Rogler G, et al: Abdominal MRI after enteroclysis or with oral contrast in patients with suspected or proven Crohn's disease. Clin Gastroenterol Hepatol 2004;2:491-497.
11 Wold PB, Fletcher JG, Johnson CD, Sandborn WJ: Assessment of small bowel Crohn's disease: noninvasive peroral CT enterography compared with other imaging methods and endoscopy - feasibility study. Radiology 2003;229:275-281.

12 Rimola J, Rodriguez S, Garcia-Bosch O, Ricart E, Pages M, Pellise M, et al: Role of 3.0-T MR colonography in the evaluation of inflammatory bowel disease. Radiographics 2009;29:701-719.

13 Schreyer AG, Ludwig D, Koletzko S, Hoffmann JC, Preiss JC, Zeitz M, et al: Updated German S3 guideline regarding the diagnosis of Crohn's disease - implementation of radiological modalities. Rofo 2010;182:116121.

14 Ajaj W, Pelster G, Treichel U, Vogt FM, Debatin JF, Ruehm SG, et al: Dark lumen magnetic resonance colonography: comparison with conventional colonoscopy for the detection of colorectal pathology. Gut 2003;52: 1738-1743.

15 Schreyer AG, Golder S, Scheibl K, Volk M, Lenhart M, Timmer A, et al: Dark lumen magnetic resonance enteroclysis in combination with MRI colonography for whole bowel assessment in patients with Crohn's disease: first clinical experience. Inflamm Bowel Dis 2005;11:388-394.

16 Schreyer AG, Hoffstetter P, Daneschnejad M, Jung EM, Pawlik M, Friedrich C, et al: Comparison of conventional abdominal CT with MR enterography in patients with active Crohn's disease and acute abdominal pain. Acad Radiol 2010;17:352-357.

17 Low RN, Sebrechts CP, Politoske DA, Bennett MT, Flores S, Snyder RJ, et al: Crohn's disease with endoscopic correlation: singleshot fast spin-echo and gadolinium-enhanced fat-suppressed spoiled gradient-echo MR imaging. Radiology 2002;222:652-660.

18 Schreyer AG, Finkenzeller T, Gossmann H, Daneschnejad M, Muller-Wille R, Schacherer D, et al: Microcirculation and perfusion with contrast-enhanced ultrasound in Crohn's disease: first results with linear contrast harmonic imaging. Clin Hemorheol Microcirc 2008;40:143-155.
19 Schreyer AG, Landfried K, Zorger N, Hoffstetter P, Ammer J, Fellner C, et al: Transmural penetration of intravenously applied $\mathrm{mi}$ crobubbles during contrast-enhanced ultrasound as a new diagnostic feature in patients with GVHD of the bowel. Bone Marrow Transplant 2011;46:1006-1011.

20 Schreyer AG, Menzel C, Friedrich C, Poschenrieder F, Egger L, Dornia C, et al: Comparison of high-resolution ultrasound and MR enterography in patients with inflammatory bowel disease. World J Gastroenterol 2011;17:1018-1025.

21 Bourreille A, Ignjatovic A, Aabakken L, Loftus EV Jr, Eliakim R, Pennazio M, et al: Role of small-bowel endoscopy in the management of patients with inflammatory bowel disease: an international OMED-ECCO consensus. Endoscopy 2009;41:618-637.

22 Desmond AN, O’Regan K, Curran C, McWilliams S, Fitzgerald T, Maher MM, et al: Crohn's disease: factors associated with exposure to high levels of diagnostic radiation. Gut 2008;57:1524-1529.

23 Zuckerman GR, Prakash C, Askin MP, Lewis BS: AGA technical review on the evaluation and management of occult and obscure gastrointestinal bleeding. Gastroenterology 2000;118:201-221.

-24 Herlinger H, Maglinte DD: Jejunal fold separation in adult celiac disease: relevance of enteroclysis. Radiology 1986;158:605-611.

-25 Soyer P, Boudiaf M, Fargeaudou Y, Dray X, Hamzi L, Vahedi K, et al: Celiac disease in adults: evaluation with MDCT enteroclysis. AJR Am J Roentgenol 2008;191:1483-1492.

26 Lohan DG, Alhajeri AN, Cronin CG, Roche CJ, Murphy JM: MR enterography of smallbowel lymphoma: potential for suggestion of histologic subtype and the presence of underlying celiac disease. AJR Am J Roentgenol 2008;190:287-293.

27 Balci NC, Noone T, Akun E, Akinci A, Klor HU: Juxtapapillary diverticulum: findings on MRI. J Magn Reson Imaging 2003;17: 487-492. 